\title{
Cholesterol is important for the entry process of porcine deltacoronavirus
}

\author{
Ji Hyun Jeon ${ }^{1} \cdot$ Changhee Lee $^{1}$ (D)
}

Received: 27 April 2018 / Accepted: 2 June 2018 / Published online: 26 July 2018

c) Springer-Verlag GmbH Austria, part of Springer Nature 2018

\begin{abstract}
The present study was conducted to examine whether cellular and/or viral cholesterol levels play a role in porcine deltacoronavirus (PDCoV) replication. Our results showed that depletion of cholesterol from cells or virions by treating them with methyl- $\beta$-cyclodextrin (M $\beta C D$ ) diminished PDCoV infection in a dose-dependent manner. The addition of exogenous cholesterol to M $\beta C D$-treated cells or virions moderately restored PDCoV infectivity. Furthermore, the pharmacological sequestration of cellular or viral cholesterol efficiently blocked both virus attachment and internalization. Taken together, the current data indicate that the cholesterol present in the cell membrane and viral envelope contributes to PDCoV replication by acting as a key component in viral entry.
\end{abstract}

Porcine deltacoronavirus (PDCoV) is a newly discovered enteric coronavirus associated with acute enteritis and intestinal damage in piglets [6, 7]. It is an enveloped, single-stranded, positive-sense RNA virus that taxonomically belongs to the genus Deltacoronavirus in the family Coronaviridae of the order Nidovirales. The virus was first discovered in pigs in Hong Kong in 2012 and subsequently, has been reported in the US, China, South Korea, Thailand, and Vietnam since 2014 [7, 9, 13, 14]. PDCoV infection results in severe villous atrophy in the small intestine, leading to watery diarrhea, vomiting, dehydration, and mortality in nursing piglets. The clinical and pathological presentations of PDCoV are indistinguishable from other swine enteric diseases caused by transmissible gastroenteritis virus (TGEV) and porcine epidemic diarrhea virus (PEDV), but there is apparently lower mortality in affected neonatal piglets [6].

Handling Editor: Sheela Ramamoorthy.

Electronic supplementary material The online version of this article (https://doi.org/10.1007/s00705-018-3967-7) contains supplementary material, which is available to authorized users.

Changhee Lee

changhee@knu.ac.kr

1 Animal Virology Laboratory, BK21 Plus KNU Creative BioResearch Group, School of Life Sciences, College of Natural Sciences, Kyungpook National University, Daegu 41566, Republic of Korea
Lipid rafts are membrane microdomains that are enriched in cholesterol, sphingolipids, and associated proteins, and are involved in the process of virus infection [1]. Cholesterol is an essential component of lipid rafts, and it plays important roles in various aspects of the virus life cycle, especially viral entry [15]. In particular, the successful entry of enveloped viruses including many coronaviruses requires the presence of cholesterol in either the viral and cellular membranes or both $[3,5,10-12,16]$. However, the potential relationship between cholesterol and PDCoV replication remains undetermined. Therefore, in this study, we investigated the necessity of cholesterol and the mechanism by which it acts in PDCoV infection.

Swine testicle (ST) cells were cultured in alpha minimum essential medium ( $\alpha$-MEM, Invitrogen, Carlsbad, CA) supplemented with $5 \%$ fetal bovine serum (FBS, Invitrogen). PDCoV strain KNU16-07 was propagated in ST cells in virus growth medium [ $\alpha$-MEM supplemented with antibiotic-antimycotic solutions, $10 \mathrm{mM}$ HEPES (Invitrogen), and $5 \mu \mathrm{g} / \mathrm{ml}$ of trypsin] as described previously [4]. M $\beta C D$ and water-soluble cholesterol were purchased from Sigma (St. Louis, MO) and dissolved in distilled water (DW) and ethanol, respectively. The cytotoxic effects of these compounds on ST cells were analyzed using a 3-(4,5-dimethylthiazol2-yl)-2,5-diphenyltetrazolium bromide (MTT) assay (Sigma) as described previously [5]. PDCoV N protein-specific monoclonal antibody (MAb) KDN 4-1 used in this study was described previously [4]. 
ST cells were pretreated with $\mathrm{M} \beta \mathrm{CD}$ or DW for $1 \mathrm{~h}$, mock infected or infected with PDCoV at a multiplicity of infection of 1 , and then cultivated in virus growth medium supplemented with $\mathrm{M} \beta C D$ or vehicle at the desired concentrations unless otherwise indicated. The M $\beta C D$-treated and virus-infected ST cells were analyzed by immunofluorescence assay (IFA) and fluorescence-activated cell sorting (FACS) as described previously [4, 5]. The culture supernatants were also collected at $24 \mathrm{~h}$ post-infection (hpi). The PDCoV titer was measured by limiting dilution on ST cells in duplicate, and $50 \%$ tissue culture infectious dose $\left(\mathrm{TCID}_{50}\right)$ per $\mathrm{ml}$ was calculated as described previously [4]. For cholesterol replenishment, ST cells were first preincubated with vehicle (DW) or $\mathrm{M} \beta C D$ at various final concentrations for $1 \mathrm{~h}$, supplemented with $10 \mu \mathrm{g} / \mathrm{ml}$ exogenous cholesterol or $0.1 \%(\mathrm{v} / \mathrm{v})$ ethanol as a vehicle control in cell culture medium for $1 \mathrm{~h}$ and then inoculated with PDCoV. In parallel, the cellular cholesterol content was determined using a Cholesterol Cell-Based Detection Assay Kit (Cayman Chemical, Ann Arbor, MI) according to the manufacturer's instructions [5].

To remove cholesterol from viral membranes, viral suspensions were treated with $\mathrm{M} \beta \mathrm{CD}$ at various concentrations at $37{ }^{\circ} \mathrm{C}$ for $1 \mathrm{~h}$ followed by ultracentrifugation to remove the $\mathrm{M} \beta \mathrm{CD}$ as described previously [5]. For cholesterol replenishment, virus suspensions were mock treated or treated with $\mathrm{M} \beta \mathrm{CD}$ at $37^{\circ} \mathrm{C}$ for $1 \mathrm{~h}$ and then supplemented with or without $100 \mu \mathrm{g} / \mathrm{ml}$ exogenous cholesterol for $1 \mathrm{~h}$ followed by ultracentrifugation as described previously [5]. In addition, the virion cholesterol content was determined by fluorescence intensity analysis using filipin III (Cayman Chemical) with a SPARK 10M multimode microplate reader (TECAN, Männedorf, Switzerland) as described previously [5]. All statistical analyses were performed using Student's $t$ test, and $P$-values of less than 0.05 were considered statistically significant.

According to the results of an MTT assay, none of the doses of $\mathrm{M} \beta \mathrm{CD}$ and cholesterol employed in the present study had an adverse effect on cell viability (Supplementary Fig. S1). ST cells were pretreated with M $\beta C D$ at concentrations of 1 and $3 \mathrm{mM}$, or with DW as a vehicle control, for 1 $\mathrm{h}$ prior to infection. Viral production was initially measured by monitoring cytopathic effects (CPE) and was later confirmed by IFA using the anti-N protein MAb. M $\beta C D$ (the cholesterol-sequestering compound) dramatically reduced virus-induced $\mathrm{CPE}$ and expression of the $\mathrm{PDCoV}$ gene in a dose-dependent manner (Supplementary Fig. S2A). Based on the quantification of the $\mathrm{N}$ protein staining results, the proportion (\%) of virus-infected cells was noticeably attenuated by $\mathrm{M} \beta \mathrm{CD}$ treatment. There was an approximate maximum of $~ 95 \%$ inhibition of both viruses in response to the application of $3 \mathrm{mM} \mathrm{M} \beta C D$ (Fig. 1A). Treating cells with $3 \mathrm{mM} \mathrm{M} \beta \mathrm{CD}$ at 0 and $1 \mathrm{hpi}$ also resulted in an approximate $\sim 85 \%$ decrease in PDCoV production, whereas exposure to the compound at 2-24 hpi had no significant inhibitory effect on infectivity compared with the control levels (Supplementary Fig. S3). These results demonstrate that M $\beta C D$ must be present pre-infection or at an early stage of viral infection to exert its antiviral effect as a cellular cholesterol depletion reagent. We also determined viral yields during the pharmacological depletion of cellular cholesterol. As illustrated in Fig. 1B, M $\beta C D$ inhibited the growth of viral progeny in a dose-dependent manner. The peak viral titer in the vehicletreated control was $10^{8.45} \mathrm{TCID}_{50} / \mathrm{ml}$ in the vehicle-treated control, whereas the addition of $3 \mathrm{mM} \mathrm{M} \beta C D$ reduced the titer of PDCoV to $10^{6.45} \mathrm{TCID}_{50} / \mathrm{ml}$ (representing a 2-log reduction compared with the control level). Taken together, our data indicate that depleting cholesterol from target cells efficiently suppresses PDCoV replication.

Next, we examined whether cholesterol replenishment restored PDCoV infectivity in M $\beta C D$-treated cells. The addition of exogenous cholesterol to M $\beta C D$-treated and virus-infected cells significantly reversed the antiviral activity of $\mathrm{M} \beta \mathrm{CD}$. Although incubation with $\mathrm{M} \beta \mathrm{CD}$ alone greatly reduced PDCoV production to $59 \%$ and $29 \%$ at concentrations of $1 \mathrm{mM}$ and $3 \mathrm{mM}$, respectively, supplementation with exogenous cholesterol significantly increased PDCoV production to $64 \%$ and $49 \%$, respectively, at the same concentrations of $\mathrm{M} \beta \mathrm{CD}$ (Fig. 1C). Consistently, cholesterol replenishment resulted in an increase in the virus yield comparable to that of the vehicle control (Fig. 1D). To verify the importance of cellular cholesterol, we then investigated alterations in cellular cholesterol content in cells treated with $\mathrm{M} \beta \mathrm{CD}$ in the absence or presence of exogenous cholesterol using filipin III, which is a fluorescent polyene antibiotic that binds to cholesterol. In accordance with the lower intensity of CPE, cellular cholesterol levels specifically decreased in virus-infected $\mathrm{M} \beta C D$-treated cells compared with those in virus-infected non-treated cells. Supplementation with exogenous cholesterol markedly elevated the cholesterol level and CPE production in virus-infected M $\beta C D$-treated cells (Fig. 1E). Altogether, the data reveal that cellular cholesterol plays a pivotal role in PDCoV infection.

To analyze whether virion-associated cholesterol is required for PDCoV infection, the virus was mock-treated or treated with $\mathrm{M} \beta \mathrm{CD}$ at concentrations of 1 and $3 \mathrm{mM}$ prior to inoculation, and the infectivity was investigated using virological methods. Similar to, but less effective (only $3 \mathrm{mM}$ ) than the effect of cellular cholesterol depletion, the removal of cholesterol from virions resulted in a significant reduction in the replication of PDCoV (a reduction of almost $60 \%$ at the highest concentration used) (Fig. 2A; Supplementary Fig. S2B). Furthermore, the PDCoV titer was reduced to $10^{7.20} \mathrm{TCID}_{50} / \mathrm{ml}$ following treatment of the virus with 3 mM M $\beta C D$ (representing a more than 1-log reduction compared with the control level) (Fig. 2B). 

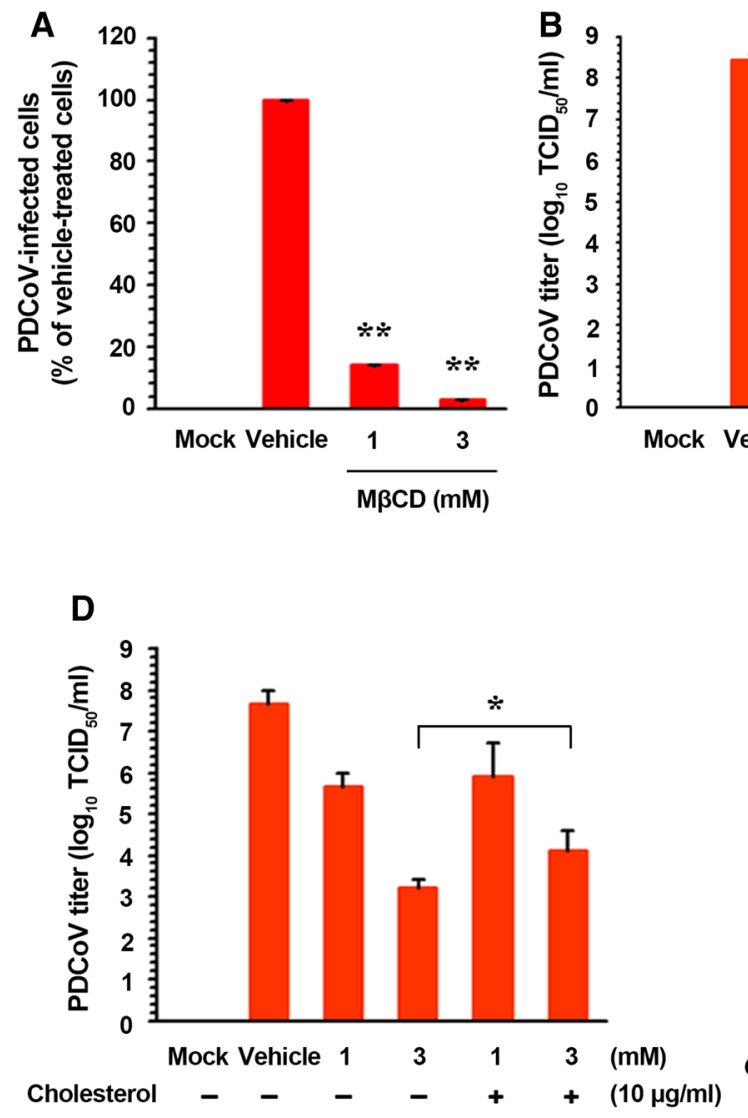

Fig. 1 Effects of cellular cholesterol depletion and replenishment on the replication of PDCoV. (A) PDCoV infection efficiency after cholesterol depletion from the cell membrane. ST cells were preincubated with $\mathrm{M} \beta \mathrm{CD}$ at the indicated concentrations for $1 \mathrm{~h}$ and were mock-infected or infected with PDCoV. Viral production in the presence of $\mathrm{M} \beta C D$ was calculated by measuring the percentage of cells expressing PDCoV N proteins using flow cytometry. (B) Viral progeny production by cellular cholesterol depletion. ST cells pretreated with $\mathrm{M} \beta \mathrm{CD}$ were infected with PDCoV and maintained in the presence of $\mathrm{M} \beta \mathrm{CD}$. At $24 \mathrm{hpi}$, virus culture supernatants were collected and the PDCoV titer was determined. (C and D) PDCoV infection efficiency after cholesterol depletion and replenishment from the cell membrane. ST cells were preincubated with $\mathrm{M} \beta \mathrm{CD}$ with (+) or without (-) exogenous cholesterol and infected with PDCoV in the presence or absence of $\mathrm{M} \beta \mathrm{CD}$ and/or exogenous cholesterol as indicated.

To verify whether the effect of virion cholesterol depletion was reversible, exogenous cholesterol was added to viral suspensions pretreated with $\mathrm{M} \beta C D$. PDCoV infection decreased to $60 \%$ in the presence of $\mathrm{M} \beta \mathrm{CD}$ alone at $3 \mathrm{mM}$, whereas virus production increased to $71 \%$ at the same concentration of $\mathrm{M} \beta \mathrm{CD}$ when exogenous cholesterol was added (Fig. 2C). Consequently, the reduced viral titer following $\mathrm{M} \beta \mathrm{CD}$ treatment was restored to values close to those observed in vehicle-treated virus-infected cells (Fig. 2D). To confirm these results, we measured the content of viral cholesterol following treatment with $\mathrm{M} \beta \mathrm{CD}$ alone, and following treatment with $\mathrm{M} \beta \mathrm{CD}$ and cholesterol replenishment. As shown in Fig. 2E, the viral cholesterol levels were
Viral infectivity was determined by measuring the percentage of cells expressing PDCoV N proteins using FACS analysis (C) and by virus titration (D). (E) Cholesterol content determination after cholesterol depletion and replenishment from the cell membrane. ST cells were preincubated with $\mathrm{M} \beta \mathrm{CD}$ with (+) or without (-) exogenous cholesterol and infected with PDCoV in the presence or absence of M $\beta C D$ and/or exogenous cholesterol as indicated. Virus-specific CPE were observed daily and photographed at 24 hpi using a fluorescent/brightfield microscope at a magnification of $200 \times$ (first row of panels). For immunostaining, infected cells were fixed at $24 \mathrm{hpi}$ and incubated with a cholesterol-binding, fluorescent antibiotic, Filipin III (second row of panels). The cells were examined using a fluorescent microscope at $200 \times$ magnification. The values shown are the means of three independent experiments, and error bars represent standard deviations. *, $P=0.001-0.05 ; * *, P<0.001$

significantly reduced in the $\mathrm{M} \beta \mathrm{CD}$-treated viruses compared with those in the vehicle-treated viruses. However, exogenous cholesterol restored the cholesterol values of the viral membranes to close to those determined prior to $\mathrm{M} \beta \mathrm{CD}$ treatment. Our results reveal that the cholesterol contents of both the cell membrane and the viral envelope are relevant to $\mathrm{PDCoV}$ infection in vitro.

To determine at what point pharmacological depletion of cellular or viral cholesterol directly influences PDCoV entry, we assessed two stages of viral entry (attachment and penetration) using binding and internalization assays after treatment of the target cells or virions with $M \beta C D$ as described previously [5]. We treated cells with $\mathrm{M} \beta \mathrm{CD}$ 

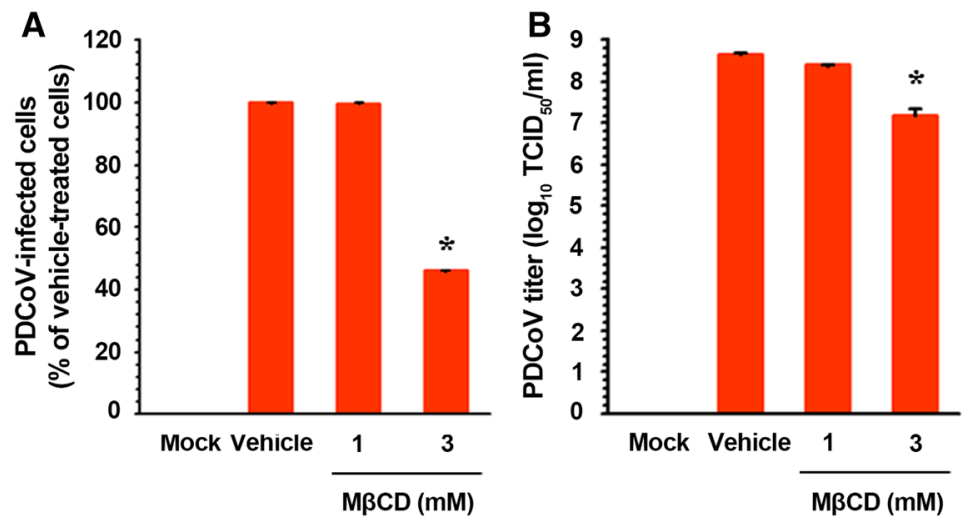

C

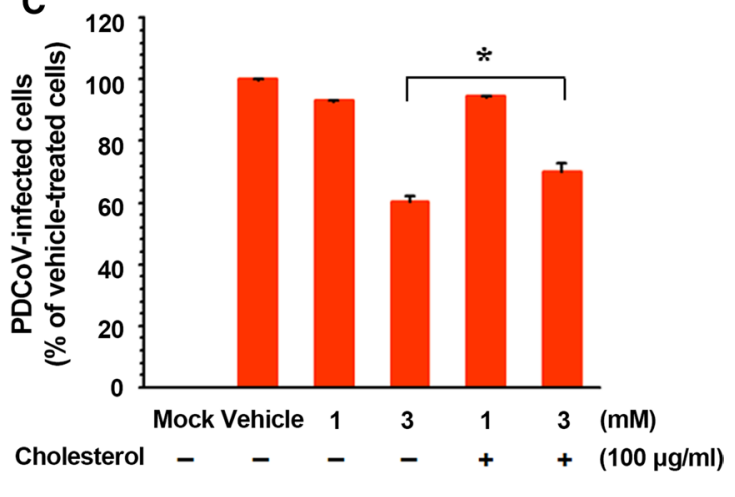

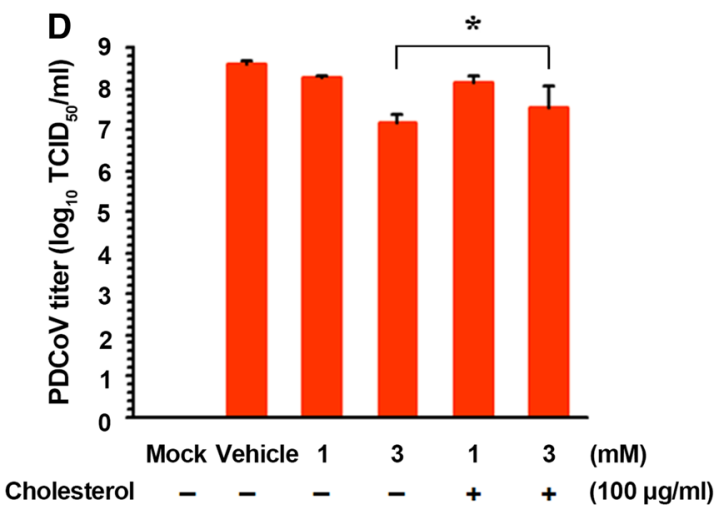

Fig. 2 Effects of viral cholesterol depletion and replenishment on the replication of PDCoV. (A) PDCoV infection efficiency after cholesterol depletion from the virus envelope. PDCoV suspensions were treated with $\mathrm{M} \beta \mathrm{CD}$ to remove cholesterol in the viral envelope, followed by ultracentrifugation, and the purified PDCoV was used to infect fresh ST cells. Virus infectivity was determined by measuring the percentage of cells expressing $\mathrm{N}$ proteins of PDCoV using FACS analysis. (B) Viral progeny production after viral cholesterol depletion. Virus culture supernatants were collected at the same timepoint, and PDCoV titers were determined. (C and D) PDCoV infection efficiency after cholesterol depletion and replenishment from the viral envelope. PDCoV suspensions were treated with $\mathrm{M} \beta \mathrm{CD}$ with

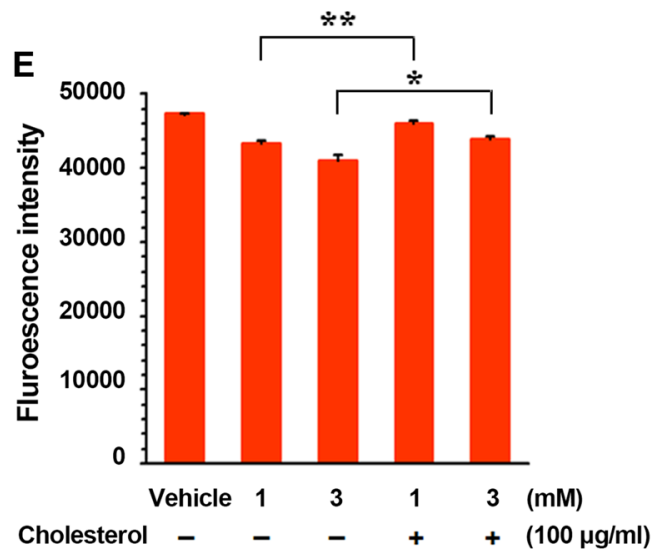

(+) or without (-) exogenous cholesterol, followed by ultracentrifugation and infection. Virus infectivity was determined by measuring the percentage of cells expressing PDCoV $\mathrm{N}$ proteins using FACS (C) and by virus titration (D). (E) Cholesterol content determination after cholesterol depletion and replenishment from the viral envelope. PDCoV suspensions were incubated with $\mathrm{M} \beta \mathrm{CD}$ with (+) or without (-) exogenous cholesterol, followed by ultracentrifugation. Virion cholesterol contents were determined using filipin III, and fluorescence intensity was measured with a fluorescence microplate reader. The values shown are the means of three independent experiments, and error bars represent standard deviations. *, $P=0.001-0.05$; **, $P<0.001$

both the cell membrane and viral envelope are indispensable for the PDCoV entry process.

In conclusion, our findings indicate that optimal infectivity of PDCoV requires cholesterol in the cell membrane and virus envelope, and that this is critical for the entry of PDCoV. Many coronaviruses exploit cholesterol, which is present in the viral envelope and/or the cell membrane for maximal virus entry. Only cellular cholesterol is essential for the entry of some coronaviruses including mouse hepatitis virus [2], severe acute respiratory syndrome coronavirus [8], human coronavirus 229E [10], avian infectious bronchitis virus [3], type II feline coronavirus (FCoV) [16], and PEDV [5]. However, as with PDCoV in the present study, canine coronavirus, type $1 \mathrm{FCoV}$, and TGEV 


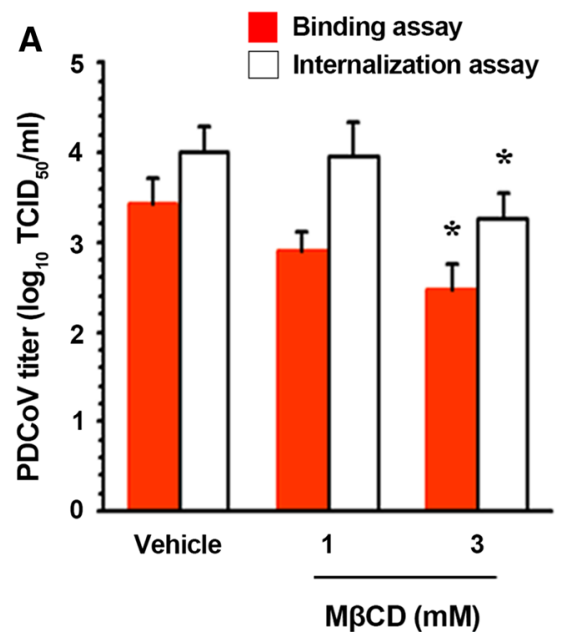

Fig. 3 Effects of cellular (A) or viral (B) cholesterol depletion on virus entry. (A) ST cells were pretreated M $\mathrm{SCD}$ infected with PDCoV at $4{ }^{\circ} \mathrm{C}$ for $1 \mathrm{~h}$. After washing with cold PBS, infected cells were maintained in the presence or absence of $\mathrm{M} \beta \mathrm{CD}$, either at $4{ }^{\circ} \mathrm{C}$ (binding) or $37^{\circ} \mathrm{C}$ (internalization), for $1 \mathrm{~h}$. The virus-infected cells maintained at $37{ }^{\circ} \mathrm{C}$ were further treated with proteinase $\mathrm{K}$ at $37^{\circ} \mathrm{C}$. The infected cells were then serially diluted and plated onto fresh target

require cholesterol in both the target cell membrane and the viral envelope $[11,12,16]$. Considering previous and present studies, the cholesterol dependence of infection differs among coronaviruses. Because the PDCoV receptor has not yet been identified, we were unable to examine whether cholesterol dependence is quantitatively related to the presence of a hitherto-unidentified receptor. Future research should address the question of whether cholesterol facilitates PDCoV entry through interactions between the viral spike protein and the cellular receptor. Nevertheless, we propose that both cellular and viral cholesterol are key players in the attachment and penetration stages of PDCoV entry. However, cholesterol depletion from the cell or virus consistently resulted in the reduction, but not the elimination, of viral infectivity. This indicates that viral entry may occur when there are low levels of cholesterol, but increased cholesterol content in both parts makes the process more efficient. Although our analysis did not elucidate the mechanism by which cholesterol promotes PDCoV entry, we assume that cholesterol-dependent viral entry is closely connected to maintaining the lipid raft structure and/or biological membrane fluidity. Impeding virus entry is a viable antiviral strategy because it likely acts on extracellular targets, thereby limiting cell damage. Therefore, PDCoV could be used as a surrogate model for testing emerging coronavirus antiviral therapies. Since no treatments or vaccines are currently available for PDCoV, the results presented here indicate that molecules that disrupt viral and/or cell cholesterol, and interfere with cholesterol function during viral entry, may provide an excellent

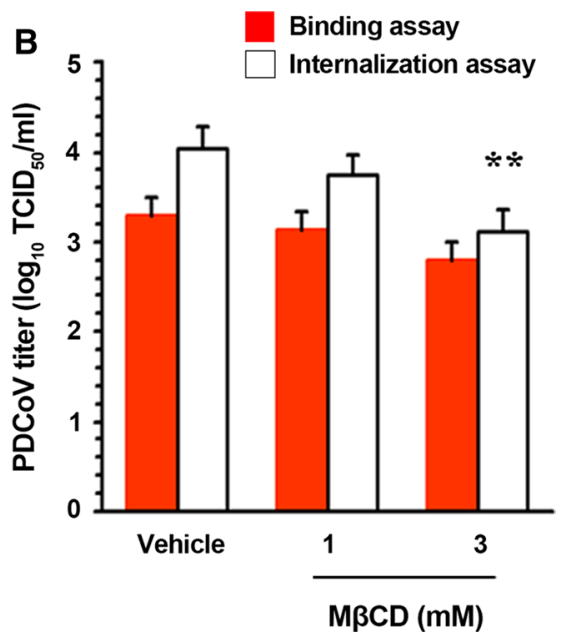

cells. At 2 days post-incubation, bound or internalized viruses were titrated. (B) PDCoV was treated with $\mathrm{M} \beta \mathrm{CD}$ and ultracentrifuged, and the purified virus was used to infect ST cells in the absence of $\mathrm{M} \beta \mathrm{CD}$ to measure bound or internalized viruses exactly as described above. The results are expressed as the mean values from three independent experiments performed in triplicate, and error bars represent standard deviations. *, $P=0.001-0.05 ; * *, P<0.001$

therapeutic option for the treatment of coronavirus infection in humans and animals.

Funding This research was supported by the Basic Science Research Program through the National Research Foundation of Korea (NRF) funded by the Ministry of Education (NRF-2015R1D1A1A09057406).

\section{Compliance with ethical standards}

Conflict of interest The authors declare that they have no conflict of interest.

Ethical approval This article does not contain any studies with animals performed by any of the authors.

\section{References}

1. Barman S, Nayak DP (2007) Lipid raft disruption by cholesterol depletion enhances influenza A virus budding from MDCK cells. J Virol 81:12169-12178

2. Choi KS, Aizaki H, Lai MM (2005) Murine coronavirus requires lipid rafts for virus entry and cell-cell fusion but not for virus release. J Virol 79:9862-9871

3. Guo H, Huang M, Yuan Q, Wei Y, Gao Y, Mao L, Gu L, Tan YW, Zhong Y, Liu D, Sun S (2017) The important role of lipid raft-mediated attachment in the infection of cultured cells by coronavirus infectious bronchitis virus beaudette strain. PLoS One 12:e0170123

4. Jang G, Kim SH, Lee YG, Kim S, Lee DS, Lee KK, Lee C (2018) Isolation and characterization of a Korean porcine deltacoronavirus strain KNU16-07. J Vet Sci 19:586-590

5. Jeon JH, Lee C (2017) Cellular cholesterol is required for porcine nidovirus infection. Arch Virol 162:3753-3767 
6. Jung K, Hu H, Saif LJ (2016) Porcine deltacoronavirus infection: etiology, cell culture for virus isolation and propagation, molecular epidemiology and pathogenesis. Virus Res 226:50-59

7. Lee S, Lee C (2014) Complete genome characterization of Korean porcine deltacoronavirus strain KOR/KNU14-04/2014. Genome Announc 2:e01191-14

8. Li GM, Li YG, Yamate M, Li SM, Ikuta K (2007) Lipid rafts play an important role in the early stage of severe acute respiratory syndrome-coronavirus life cycle. Microbes Infect 9:96-102

9. Marthaler D, Jiang Y, Collins J, Rossow K (2014) Complete genome sequence of strain SDCV/USA/Illinois121/2014, a porcine deltacoronavirus from the United States. Genome Announc 2:e0218-14

10. Nomura R, Kiyota A, Suzaki E, Kataoka K, Ohe Y, Miyamoto K, Senda T, Fujimoto T (2004) Human coronavirus 229E binds to $\mathrm{CD} 13$ in rafts and enters the cell through caveolae. J Virol 78:8701-8708

11. Pratelli A, Colao V (2015) Role of the lipid rafts in the life cycle of canine coronavirus. J Gen Virol 96:331-337
12. Ren X, Glende J, Yin J, Schwegmann-Wessels C, Herrler G (2008) Importance of cholesterol for infection of cells by transmissible gastroenteritis virus. Virus Res 137:220-224

13. Saeng-Chuto K, Lorsirigool A, Temeeyasen G, Vui DT, Stott CJ, Madapong A, Tripipat T, Wegner M, Intrakamhaeng M, Chongcharoen W, Tantituvanont A, Kaewprommal P, Piriyapongsa J, Nilubol D (2017) Different lineage of porcine deltacoronavirus in Thailand, Vietnam and Lao PDR in 2015. Transbound Emerg Dis 64:3-10

14. Song D, Zhou X, Peng Q, Chen Y, Zhang F, Huang T, Zhang T, Li A, Huang D, Wu Q, He H, Tang Y (2015) Newly emerged porcine deltacoronavirus associated with diarrhoea in swine in China: identification, prevalence and full-length genome sequence analysis. Transbound Emerg Dis 62:575-580

15. Suzuki T, Suzuki Y (2006) Virus infection and lipid rafts. Biol Pharm Bull 29:1538-1541

16. Takano T, Satomi Y, Oyama Y, Doki T, Hohdatsu T (2016) Differential effect of cholesterol on type I and II feline coronavirus infection. Arch Virol 161:125-133 